\title{
Hypoxic-Ischemic Injury in the Developing Brain: The Role of Reactive Oxygen Species Originating in Mitochondria
}

\author{
Vadim S. Ten ${ }^{1,2,3}$ and Anatoly Starkov ${ }^{2}$ \\ ${ }^{1}$ Department of Pediatrics, Columbia University, NY, USA \\ ${ }^{2}$ Department of Neurology and Neuroscience, Cornell University, NY, USA \\ ${ }^{3}$ Division of Neonatology, Department of Pediatrics, Morgan Stanley Children's Hospital of New York, 3959 Broadway, BHN 1201, \\ New York, NY 10032, USA \\ Correspondence should be addressed to Vadim S. Ten, vt82@columbia.edu
}

Received 6 September 2011; Revised 12 November 2011; Accepted 22 November 2011

Academic Editor: Robin L. Haynes

Copyright ( $) 2012$ V. S. Ten and A. Starkov. This is an open access article distributed under the Creative Commons Attribution License, which permits unrestricted use, distribution, and reproduction in any medium, provided the original work is properly cited.

\begin{abstract}
Mitochondrial dysfunction is the most fundamental mechanism of cell damage in cerebral hypoxia-ischemia and reperfusion. Mitochondrial respiratory chain (MRC) is increasingly recognized as a source for reactive oxygen species (ROS) in the postischemic tissue. Potentially, ROS originating in MRC can contribute to the reperfusion-driven oxidative stress, promoting mitochondrial membrane permeabilization. The loss of mitochondrial membranes integrity during reperfusion is considered as the major mechanism of secondary energy failure. This paper focuses on current data that support a pathogenic role of ROS originating from mitochondrial respiratory chain in the promotion of secondary energy failure and proposes potential therapeutic strategy against reperfusion-driven oxidative stress following hypoxia-ischemia-reperfusion injury of the developing brain.
\end{abstract}

\section{Introduction}

Perinatal hypoxic-ischemic (HI) brain injury is one of the most common causes of severe neurological handicap in children. Estimated life-time costs to support children with cerebral palsy, a common outcome of HI brain injury in neonates, reached 11.5 billion dollars in 2003 [1]. Unfortunately, our understanding the mechanisms of the $\mathrm{HI}$ brain injury is not deep enough for the development of mechanism-targeted therapeutic interventions in this disease. Even therapeutic mechanisms of post-HI cerebral hypothermia (the only clinically proven neuroprotective strategy) are still not well defined which precludes an optimal use of this potentially powerful strategy.

Physiologically, HI brain injury could be defined as an acute oxygen and nutrients deprivation to the brain caused by a collapse of cerebral circulation. Hypoxia-ischemia results in severe cellular bioenergetics failure, and if cerebral circulation is not restored, then the brain death is unpreventable. However, if the cerebral circulation is restored for example, as a result of successful resuscitation, then cerebral reperfusion ensures with a full or partial brain recovery. Unfortunately, the same reperfusion can also contribute to the propagation of brain injury initiated by the $\mathrm{HI}$ insult. This implies that HI brain injury as a disease, consists of two fundamental pathophysiological events: hypoxiaischemia and reperfusion. During hypoxia-ischemia and reperfusion mitochondrial dysfunction plays a fundamental role in brain injury. It is now recognized that not only mitochondrial failure to generate ATP during ischemia, but the generation of oxidative radicals and the release of proapoptotic proteins during reperfusion contribute to the cellular damage. The leading molecular mechanisms responsible for the evolution of cell damage and repair during reperfusion change at different timepoints following HI insult (Figure 1). A critical upstream mechanisms to consider in the management of HI brain injury are those linked to an oxidative stress [2]. Therefore, already at the initiation of resuscitation/reperfusion an attempt should be made to limit the reoxygenation-driven burst in generation of reactive oxygen species (ROS) in order to alleviate the severity of oxidative damage to the HI brain. 


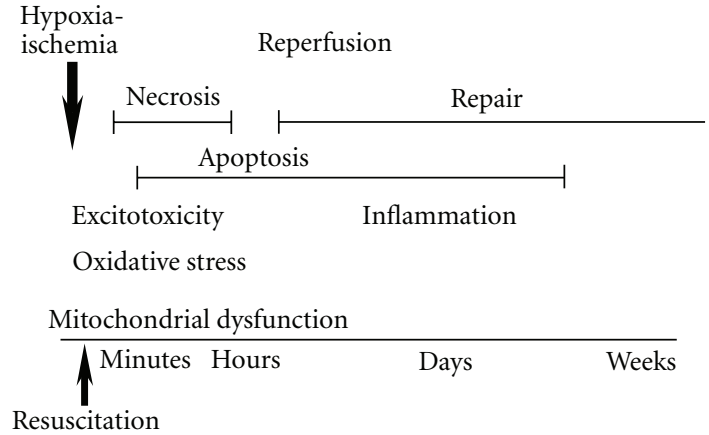

Figure 1: The evolution and major mechanisms of hypoxicischemic brain injury. Arrows indicate $\mathrm{HI}$ insult and resuscitation (reperfusion), two fundamental events that cause cerebral damage. Different mechanisms may take a lead in the evolution of brain injury: initiated by the bioenergetics mitochondrial dysfunction, cellular injury is driven by excitotoxicity and oxidative stress, followed by the neuroinflammation. The paper is focused on the proximal to the index event mechanism, an oxidative stress and the role of mitochondrial generation of ROS (see text), modified from [2].

\section{HI and Resuscitation}

It is known that reintroduction of the oxygen to ischemic tissue potentiates oxidative injury. An initial attempt to limit formation of ROS could be made by judicious use of oxygen during resuscitation. Not too long ago, in 2000 the use of $100 \%$ oxygen was indisputably recommended for the initiation of resuscitation in all depressed infants [3]. Now neonatologists have tempered their enthusiasm for the use of pure oxygen in neonatal resuscitation. Several clinical trials showed that in the majority of depressed infants the goal of resuscitation, an immediate survival, could be achieved with the use of room air, as effectively as with the use of $100 \%$ oxygen [4-6]. Oxygen is indispensable component of ROS. Therefore, regardless of the primary mechanisms of ROS generation during reperfusion, a switch from a routine use of $100 \%$ oxygen to the room air at the initiation of neonatal resuscitation, potentially, should limit the severity of an oxidative stress. Indeed, Vento et al. reported a significantly lower level of circulating markers of oxidative stress in neonates resuscitated with the room air (RA) compared to infants resuscitated with the $100 \%$ oxygen [7]. However, it remains to be determined to what extent the use of RA in the resuscitation of infants with HI brain injury attenuates an oxidative damage to the brain. Numerous animal studies clearly demonstrated that hyperoxic re-oxygenation maintained for 30-60 minutes of initial reperfusion was detrimental for neurological outcome in asphyxiated pigs and rodents [8-10]. The use of the $100 \%$ oxygen in these animals was strongly associated with exacerbation of an oxidative stress in the brain [8]. Of note, however, the hyperoxic resuscitation in these studies was used for 30-60 minutes. At these time-points of reperfusion a full restoration of systemic circulation was already achieved and this resulted in extreme hyperoxemia. Because the primary goal of resuscitation is the return of spontaneous circulation (ROSC), experiments in which the hyperoxic resuscitation is applied beyond the time-point of the ROSC have limited translational importance for the resuscitation science. However, the references cited above do provide an important translational message for the postresuscitation medical care: All efforts should be made to avoid hyperoxemia in reperfusion.

Although, normoxic resuscitation has been shown to be effective in the majority of infants, it is still undetermined whether the use of RA in the resuscitation of severely (a complete circulatory arrest) asphyxiated infants is as effective as the use of $100 \%$ oxygen in achieving ROSC. After a prolonged (25 minutes) cardiopulmonary arrest in mature pigs, the resuscitation with the use of positive pressure ventilation significantly improved the rate of sustained ROSC and cardiac output only if the resuscitation was supplemented with hyperbaric $\left(\sim 400 \% \mathrm{O}_{2}\right)$ re-oxygenation [11]. In contrast, following a brief (one minute) cardiac arrest a cardio-pulmonary resuscitation with the use of RA or $100 \% \mathrm{O}_{2}$ resulted in similar rates of ROSC in neonatal pigs $[12,13]$. These data suggest that the duration of circulatory arrest may determine whether positive pressure ventilation needs supplementation with $100 \% \mathrm{O}_{2}$ to enhance the rate of ROSC. It is critical to understand that no attempts should be made to attenuate a reperfusion-driven oxidative stress at the expense of the efficacy of resuscitation.

Overall, current data suggest that the use of room air in resuscitation reduces the severity of oxidative stress in the majority of depressed infants at risk for HI brain injury. The simplicity of this approach (restriction of oxygen availability for the formation of ROS), however, underscores our incomplete understanding the mechanisms initiating an oxidative injury to the HI brain. Interestingly, Matsiukevich et al. showed that in neonatal mice subjected to a lethal HI insult evidenced by a complete circulatory collapse, hyperoxic resuscitation limited to the time ( 2 minutes) needed to achieve a sustained ROSC was not associated with exacerbation of reperfusion-driven acceleration in the rate of ROS emission from isolated brain mitochondria [14]. However, it is yet to be clarified whether ROS originating from mitochondria at the onset and during reperfusion cause an oxidative injury to the HI brain. To date, it is still unclear what are sources of pathogenic oxidative radicals in the HI brain, how to enhance antioxidative mechanisms and what are those mechanisms of injury which are initiated or exacerbated by the ROS.

\section{Potential Sources of Reactive Oxygen Species in HI Injury to the Developing Brain}

The evolution of ischemic brain injury following restoration of oxygen and nutrient delivery is a paradoxical biological phenomenon. Although, it is clear that without reperfusion/reoxygenation an ischemic tissue does not survive, maladaptive metabolic changes induced by ischemia predispose cell to dysfunction and death upon reperfusion/reoxygenation. The central role in this phenomenon was assigned to ROS, which can be formed only in the presence of $\mathrm{O}_{2}$. Therefore, an oxidative damage occurs 
mostly upon reintroduction of $\mathrm{O}_{2}$ to the ischemic tissue. In the immature brain antioxidant system is underdeveloped which limits inactivation of some ROS and in particular, hydrogen peroxide (reviewed in [2]). The latter is perhaps the most important tissue-damaging ROS species due to its relative stability and the ability to cross lipid membranes. For example, upregulation of $\mathrm{Cu} / \mathrm{Zn}$ superoxide dismutase (enzyme which converts superoxide into $\mathrm{H}_{2} \mathrm{O}_{2}$ ) increased, rather than decreased the extent of $\mathrm{HI}$ brain injury in neonatal rats [15]. This was associated with elevated level of $\mathrm{H}_{2} \mathrm{O}_{2}$ in the brain. In contrast, transgenic mice overexpressing glutathione peroxidase (enzyme which detoxifies $\mathrm{H}_{2} \mathrm{O}_{2}$ into $\mathrm{H}_{2} \mathrm{O}$ ) were markedly protected against $\mathrm{HI}$ insult [16]. What is the origin of this $\mathrm{H}_{2} \mathrm{O}_{2}$ ? What are the major sources of oxidative radicals responsible for an oxidative brain damage in HI? In an elegant study, Abramov and coauthors have identified three distinct ROS generating systems during simulated HI insult (oxygen-glucose deprivation (OGD)) and reperfusion in cultured neurons mitochondrial respiratory chain (MRC), xanthine oxidase and NADPH oxidase [17]. MRC responds to OGD with a burst of ROS emission, which declined by the end of $\mathrm{HI}$ insult secondary to a loss of mitochondrial membrane potential. At the end of $\mathrm{HI}$ insult a second elevation in cellular ROS generation was attributable to the activity of xanthine oxidase. A third peak in ROS production was due to activity of NADPH oxidase during reperfusion. Inhibition of either NADPH oxidase or xanthine oxidase resulted in a significant neuroprotection [17]. In immature animals and humans with HI brain injury, elevated level of hypoxanthine was proposed as the evidence for a pathogenic role of xanthine oxidase $[18,19]$. However, an inhibition of xanthine oxidase with oxypurinol or allopurinol failed to reduce lipid peroxidation, and did not protect the brain in a rat model of HI injury [20] or in human neonates with perinatal HI insult [21]. Genetic or/and pharmacological inhibition of NADPH oxidase also did not exert neuroprotection in different models of perinatal HI brain injury [22]. Taken together these data challenge a pathogenic contribution of NADPH oxidase or xanthine oxidase to an oxidative brain damage following $\mathrm{HI}$ in neonates. Interestingly, Loor et al. using a model simulating $\mathrm{HI}$ reperfusion injury in cultured cardiomyocytes demonstrated that genetic overexpression of only intramitochondrial ROSscavenging enzymes, Mn-superoxide dismutase or phospholipid hydroperoxide glutathione peroxidase protected cells against reperfusion-induced death [23]. In contrast, overexpression of $\mathrm{Cu}-\mathrm{Zn}$ superoxide dismutase or catalase did not result in the protection [23].

Mitochondria are known as a major source for ROS production in the health and diseases, including brain ischemiareperfusion injury (reviewed in [24]). In mature animal models of ischemia-reperfusion injury to the brain and heart, mitochondria have been increasingly recognized as an important source for the reperfusion-driven acceleration in ROS release [24-27]. However, rapidly emerging evidence supporting a deleterious role of ROS originating in mitochondria during reperfusion are partially counterbalanced by the reports suggesting a prosurvival signaling mediated by mitochondrial ROS in the heart preconditioning ([28], reviewed in [29]) and in postischemic reperfusion [30]. In the developing brain potential deleterious or prosurvival effects of mitochondrial ROS in HI reperfusion were not studied. In the following part of this paper we discuss the experimental data obtained in the mature animal models of the brain and heart ischemia-reperfusion injury which support the primary role of mitochondrial ROS in oxidative damage.

\section{Mitochondrial ROS and HI Reperfusion Oxidative Stress}

In mature animals several studies detected a reperfusiondriven acceleration in ROS generation from mitochondria associated with oxidative damage to the postischemic heart $[25,26]$ and brain [27]. A single study showed that in neonatal mice with genetically ablated C1q component of the classical complement activation pathway, the neuroprotection and attenuation of oxidative HI brain injury were associated with the ability of $\mathrm{Clq}^{-/-}$brain mitochondria to release significantly less ROS in response to HI reperfusion, rather then with altered activation of the terminal complement complex [31]. A pathogenic contribution of ROS originating from mitochondria is supported by the data demonstrating that extrinsic or genetic enhancement of mitochondriatargeted ROS scavengers reduces the extent of injury or/and oxidative stress in animal models of ischemia-reperfusion in several organs ([32-34], reviewed in [35]). Furthermore, pharmacological inhibition of ROS generation in the mitochondrial respiratory chain (MRC) limits the extent of ischemia-reperfusion damage and the expression of markers of oxidative injury $[26,36,37]$. These data highlight MRC as a potential target for an antioxidative therapeutic strategy against $\mathrm{HI}$ brain injury. In the MRC, complex I and complex III are two major sites for ROS generation during reperfusion $[32,38]$. An inhibitory effect of ischemia on complex I has been suggested as a cause for an accelerated generation of ROS in MRC in hearts [26]. However, interpreting the data on postischemic mitochondrial ROS production might be difficult and requires an appropriate experience. The data on mitochondrial function in ischemia-reperfusion mostly were obtained in isolated mitochondria in vitro, when results depended on the choice of experimental conditions. For example, in mitochondria isolated from different organs, including neonatal mouse brain, the response to inhibition of complex I is either increase or dramatic decrease in ROS emission rates, depending upon a substrate used to donate electrons to MRC. NAD-linked substrates such as malate, glutamate, pyruvate, and so forth, invariably support an elevation in mitochondrial ROS emission following an inhibition of complex I with rotenone (Figure 2(a)). In contrast, the use of FAD-linked substrates such as for example, succinate results in robust decrease in mitochondrial ROS emission following an inhibition of complex I with rotenone (Figure 2(a)). These differences in ROS generation by MRC in response to the same complex I inhibitor are well understood and explained by the differences in the electron transport flows, supported by NAD- or FADlinked substrates (reviewed in [39]). NAD-linked substrates 
(a)

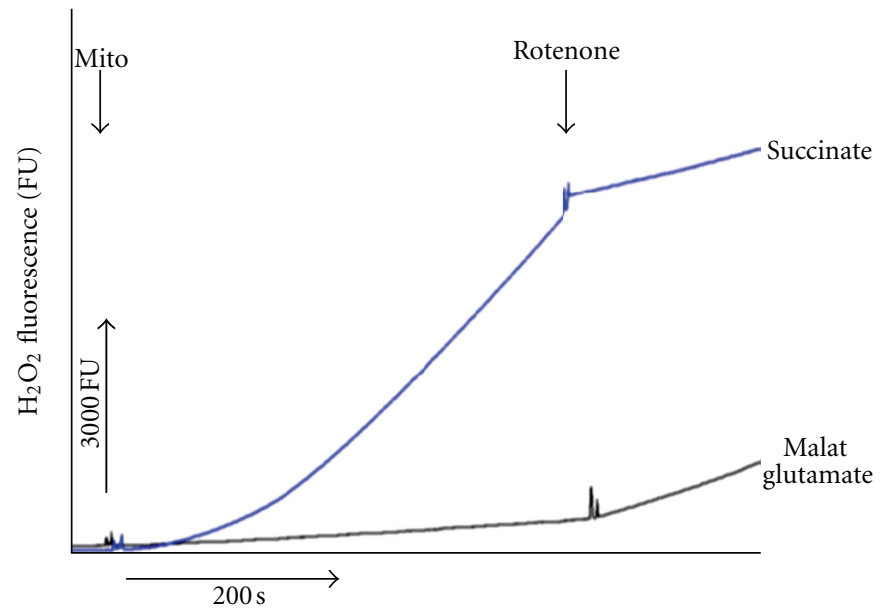

(b)

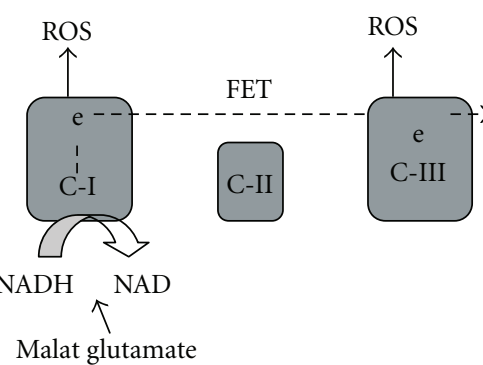

(c)

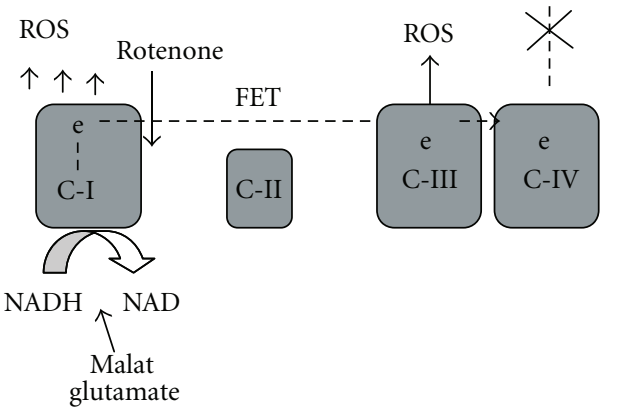

(d)

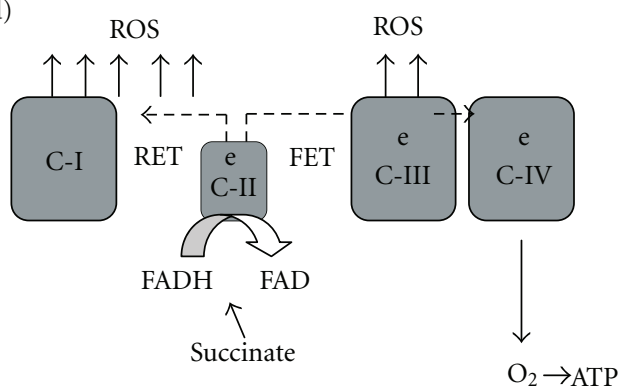

(e)

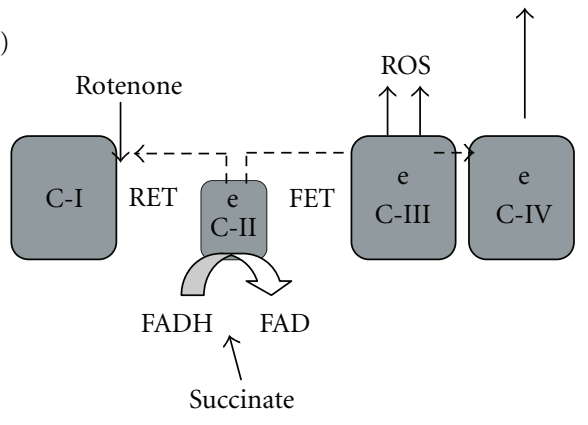

Figure 2: (a) $\mathrm{H}_{2} \mathrm{O}_{2}$ emission rate from brain mitochondria isolated from p10 naïve mouse and supported either with succinate (FAD-linked substrate) or malate-glutamate (NAD-linked substrates). Time-points when mitochondria (mito, $0.05 \mathrm{mg} / \mathrm{mL})$ or rotenone $(1 \mu \mathrm{M})$ were added are indicated. Cerebral nonsynaptic mitochondria were isolated and mitochondrial $\mathrm{H}_{2} \mathrm{O}_{2}$ fluorescence was measured using Amplexultra-red and horse radish peroxidase assay as described in [31]. (b-e), a schematic mechanism for ROS generation in MRC fueled with NAD-linked substrate, before (b) and after inhibition of complex I with rotenone (c), or FAD-linked substrate, before (d) and after rotenone supplementation (e). RET: reverse electron trasport, FET: forward electron transport.

support only forward electron transport flow (FET), from complex I- to membrane-dissolved ubiquinone-to Complex III-to cytochrome $\mathrm{c}$ and finally to oxygen through complex IV (cytochrome $c$ oxidase). During this FET, low levels of superoxide can be generated at unspecified MRC sites (likely at complex I and complex III), because some electrons accidentally escape from MRC electron carriers onto $\mathrm{O}_{2}$ (Figure 2(b)). Rotenone, pyridaben, thiobarbiturates and other complex I inhibitors interrupt FET between the complex I electron carriers and membranedissolved ubiquinone. This interruption of FET increases ROS emission from complex I (Figure 2(c)) secondary to over-reduction of electron carriers (flavin and/or FeS-center
$\mathrm{N} 2$ and complex I-bound ubiquinone) within this complex (reviewed in [40]). It also stimulates ROS emission from other sources located in the mitochondrial matrix such as for example, dihydrolipoamide dehydrogenase [41, 42], a subcomponent of pyruvate dehydrogenase and ketoglutarate dehydrogenase. This stimulation in ROS production is caused by a decrease in mitochondrial NAD/NADH ratio (as a result of inability of compelx I to oxidize NADH). On the other hand, in the mitochondria fueled with FAD-linked substrates (e.g., succinate) the main electrons flow bypasses Complex I and proceeds from the succinate dehydrogenase (Complex II) to membrane-dissolved ubiquinone, Complex III, cytochrome c, and cytochrome c oxidase. Under specific 
conditions, such as moderately elevated membrane potential and abundance of FAD-linked substrate, electron flux canand does-proceed back from complex II, ubiquinone to complex I and further to the matrix-located NAD. This is called reverse electron transport (RET) flow (Figure 2(d)). It was found that RET is associated with very high rates of ROS emission, about 100 folds greater than that obtained with NAD-linked substrates (reviewed in [39]). The major sites of ROS emission in mitochondria fueled with FAD-linked substrate are thought to be complex I and matrix-located enzymes pyruvate dehydrogenase and alpha-ketoglutarate dehydrogenase. Inhibition of complex I with rotenone or similar inhibitors interrupts RET flow and, therefore, substantially diminishes the rate of ROS emission (5-8 folds) (Figures 2(a) and 2(d)) [39]. The RET flow represents the major mechanism for ROS production by mitochondria fueled with succinate, especially in the brain and the heart [43]. It should be noted, that both FET and RET generate proton-motive force and support oxidative phosphorylation of ADP; with RET being about 30\% less efficient in terms of energy production but generating tremendously more ROS.

In vivo, under non-pathological conditions the primary electron donor for MRC in brain mitochondria are NAD-linked substrates for example, pyruvate generated in glycolysis. During ischemia-reperfusion, however, substrate availability significantly differs from that in normal cells. There are several lines of evidence to consider that at the onset of reperfusion postischemic mitochondria actively metabolize succinate. Complex I is the most sensitive among all five complexes to the reduction of the cerebral blood flow, and at the end of ischemia the activity of this complex is significantly reduced $[44,45]$. In the immature brain HI resulted in slightly ( $9 \%$ on malate-glutamate) to moderately ( $21 \%$ on pyruvate-malate) greater inhibition of mitochondrial respiration tested on NAD-linked substrates compared to that tested on the FAD-oriented substrate, succinate [46]. In mature rats, forebrain ischemia and six hours of reperfusion resulted in a significant inhibition of mitochondrial respiration tested on NAD-linked substrates. However, no significant differences from the control values were detected when the same mitochondria respired on succinate [47]. This suggests, that after brain ischemia the activity of complex II- is better preserved compared to complex I. This favors a succinate-supported respiration upon reintroduction of $\mathrm{O}_{2}$. Indeed, in the rat brain, ischemia resulted in a profound (8-10 fold) depletion of all NAD-linked substrates: pyruvate, citrate, alpha-ketoglutarate, oxaloacetate, fumarate, and malate. In contrast, the concentration of the succinate increased by $\sim 300 \%$ [48] and remained elevated at 15 minutes of reperfusion [49]. Following an acute systemic hypoxemia an oxidation of succinate and glutamate by isolated rat brain mitochondria was significantly $(>60 \%)$ increased $[50,51]$. Furthermore, it is known that succinate oxidation inhibits an oxidation of pyruvate and other NADlinked respiratory substrates, an event associated with overreduction of mitochondrial pyridine nucleotides [52]. In the heart, the level of succinate also is markedly elevated during ischemia followed by normalization within 30-60 minutes of reperfusion $[53,54]$, the time-point associated with near-full restoration of mitochondrial metabolic activity in neonatal HI reperfusion [31]. Thus, if at the initial stage of reperfusion mitochondria actively utilize succinate, then interruption of RET flow by complex I inhibiting agents should reduce ROS generation without significant changing ATP-production rate. If the RET flow-dependent production of ROS causes an oxidative damage following $\mathrm{HI}$, then inhibition of complex I recovery upon reperfusion should reduce an oxidative injury. Indeed, in rats with global cerebral ischemia an inhibition of complex I by rotenone or haloperidol significantly reduced tissue accumulation of hydroxyl radicals, resulting in nearcomplete abrogation of the reperfusion-driven surge in lipid peroxidation products [27]. Ambrosio et al. reported that inhibition of complex I with the thio-barbiturate amytal resulted in significant reduction in the level of free radicals associated with attenuation of lipid peroxidation in isolated rabbit hearts subjected to ischemia-reperfusion [25]. Our data demonstrated that inhibition of complex I with pyridaben significantly reduced cerebral infarct volume and signs of oxidative injury to the brain tissue and mitochondria following $\mathrm{HI}$ in neonatal mice [55]. In the model of cardiac arrest and reperfusion, complex I was proposed as a primary generator of ROS [56]. Taken together, these data suggest that ROS generated in complex I participate in oxidative damage to the postischemic brain and heart, making this complex a reasonable therapeutic target against oxidative stress in the early stages of reperfusion.

In addition to the complex I, complex III has been recognized as an important source for emission of ROS in ischemia and reperfusion [30, 57]. However, experiments with isolated nerve terminals revealed that only very high level of complex III inhibition $(70-80 \%)$ resulted in detectable elevation in generation of $\mathrm{H}_{2} \mathrm{O}_{2}$ [58]. Given, that after brain ischemia mitochondrial respiration on succinate was shown to be markedly better preserved compared to that tested on complex I linked substrates [47], the rationale to consider complex III as a therapeutic target in reperfusion is weak. Indeed, in mitochondria respiring on succinate the RET flow (complex I) contribute the most to ROS production. Finally, it is unrealistic to inhibit complex III without robust reduction in production of ATP which could be detrimental for the tissue recovery.

\section{The Pathogenic Mechanisms Targeted by Mitochondrial ROS in HI Reperfusion.}

Traditionally, a detrimental effect of oxidative stress is supported by evidence of structural oxidative alterations to the post-HI brain. However, it is also important to determine what specific mechanism of injury could be targeted by ROS during reperfusion. In the design of neuroprotective strategies, it is not only a source of injurious ROS, but also a particular mechanism of damage triggered/exacerbated by these ROS is important to consider. Logistically, if an oxidative stress is one of the earliest reperfusion-driven damaging events, the mechanism targeted by ROS should be in close temporal proximity to the index event.

In the ischemic brain, cells experience glutamatereceptors over-stimulation and cellular $\mathrm{Ca}^{++}$overload, which 
occurs to a markedly greater extent in the neonatal brain than in the mature CNS [59, 60]. Mitochondria actively participate in preservation of cellular $\mathrm{Ca}^{++}$homeostasis by up take of $\mathrm{Ca}^{++}$from the cytosol into mitochondrial matrix space (reviewed in [61]). However, if mitochondrial $\mathrm{Ca}^{++}$ load exceeds mitochondrial capacity to hold $\mathrm{Ca}^{++}$, then mitochondrial membranes loose their integrity via opening a channel in the inner membrane, termed the mitochondrial permeability transition pore $(\mathrm{mPTP})$. Transient and permanent opening of MPTP has been strongly considered as one of the leading mechanisms of necrotic and apoptotic cell death in the brain and other organs following ischemiareperfusion injury $([62,63]$, reviewed in [64]). It has been shown, that mitochondrial ROS can initiate an opening of $\mathrm{mPTP}$ during ischemia [22] and reperfusion $[65,66]$ even in the absence of cyclophilin-D (the only known structural component of mPTP) or $\mathrm{Ca}^{++}$overload $[67,68]$. Mitochondria-targeted antioxidant, mitoTEMPO, partially prevented $\mathrm{MPTP}$ opening and attenuated necrosis and apoptosis following simulated ischemia-reperfusion injury in cultured renal tubular cells [69]. Taken together these data suggest, that regardless of the type of the organ, ROS originating from mitochondria upon reperfusion can trigger a loss of integrity in mitochondrial inner membrane, the event suggested as the "point of no return" in propagation of cell death following HI insult.

\section{The Role of Mitochondrial Membrane Permeabilization in the HI Brain Injury}

6.1. Inner Mitochondrial Membrane Permeability Transition Pore ( $m P T P)$ and HI Injury in the Developing Brain. Independent of the developmental stage, HI insult severely inhibits mitochondrial oxidative phosphorylation. It has been shown that in immature brain, at the end of $\mathrm{HI}$ insult mitochondrial phosphorylating respiration was significantly suppressed [31, 70, 71]. Reoxygenation/reperfusion restores mitochondrial ADP-phosphorylating capacity, normalizing ATP content in the post-HI brain. However, following several hours of reperfusion mitochondria exhibit a profound decline in their ADP-phosphorylating respiration rates [31, 46], the event known as a secondary energy failure. The molecular mechanism proposed to explain the pathogenesis of secondary energy failure is opening of mPTP. mPTP renders organelles incapable of ATP production due to a loss of proton-motive force and NAD. This bioenergetics failure results in mitochondrial swelling, leading to a permeabilization of the outer mitochondrial membrane and release of pro-apoptotic proteins which eventuates in necrotic and apoptotic cell death [72-74]. It has been shown that in neonatal rats inner mitochondrial membrane opens mPTP at $0-1.5$ hours and at 6-8 hours after HI [75]. However, the pathogenic significance of $\mathrm{mPTP}$ in the reperfusion injury in the developing $\mathrm{HI}$ brain remains uncertain. For example, as opposite to adult mice, neonatal cyclophilin$\mathrm{D}$ knock-out mice were found to be susceptible to $\mathrm{HI}$ injury [76]. Earlier the same group has reported that antagonist of cyclophilin-D, cyclosporin-A did not attenuate the extent of HI brain damage in neonatal rats [77]. In contrast, using the same model Hwang et al. reported that cyclosporin-A, injected immediately after HI insult significantly protected developing brain, attenuating both necrotic and apoptotic cell death in neonatal rats [78]. Similar results were obtained in neonatal rats subjected to a mild focal cerebral ischemia-reperfusion [79]. In neonatal rats and mice subjected to a global hypoxia-ischemiareperfusion injury, a post-treatment with cyclosporine $\mathrm{A}$ markedly potentiated the neuroprotective effect of $\mathrm{Ca}^{++}$ channel antagonist, nimodipine [80]. Given, that in mature animal models of ischemia-reperfusion injury a pathogenic role for MPTP has been strongly suggested, more extensive research is needed to clarify the contribution of $\mathrm{MPTP}$ opening to cerebral HI reperfusion injury in the developing brain.

6.2. Outer Mitochondrial Membrane Pore (OMMP) and HI Injury to the Developing Brain. Following an ischemic insult mitochondrial membrane permeabilization can occur via opening of outer mitochondrial membrane pore (OMMP) induced by Bak/Bax translocation into mitochondria. This pore is thought to be primarily responsible for a release of pro-apoptotic proteins from the mitochondrial intermembrane space, leading to an apoptotic cell death [81, 82], including that induced by an oxidative stress ([83], reviewed in [84]). Importantly, in HI reperfusion injury to the developing brain Bax dependent OMMP has been suggested as a primary mechanism of injury ([76], reviewed in [85]). Developmental shift toward a priority of the Baxdependent OMMP over the cyclophylin-D dependent mPTP opening in the HI brain damage has been supported by the data obtained in cyclophilin D knock-out neonatal mice [76], as well as by neuroprotective effect of Bax-inhibiting peptide [86]. However, in contrast to a better understanding of events leading to secondary energy failure and necrotic cell death following an opening of mPTP, it is less clear how Bax/Bak mediated OMMP opening affects oxidative phosphorylation and results in secondary energy failure and necrosis. One possibility is that postischemic opening of OMMP results in a massive loss of cytochrome $\mathrm{c}$ from the inter-membrane mitochondrial space which results in secondary inhibition of oxidative phosphorylation. However, this loss of cytochrome c was not mediated by mPTP opening, and was not associated with changes in mitochondrial Bax, Bad, Bak or Bid [87]. Although, mitochondrial ROS appeared to be critical for the execution of Bax/Bak dependent apoptosis induced by anti-cancer drugs $[88,89]$, we have not found data that ROS originating in mitochondria are involved in the Bax/Bakinduced apoptosis in HI brain injury. Interestingly, oxidative stress-induced cell apoptosis clearly required the presence of ROS originating from MRC to signal mPTP opening, but this apoptosis was independent of Bax translocation [90]. The existence of two relatively independent mechanisms of mitochondrial membrane permeabilization does not exclude the contribution of each of these mechanisms in HI damage to the developing brain. Indeed, there is evidence for involvement of cyclophilin D dependent mPTP opening in the Bax-driven cytochrome $c$ release in the isolated mitochondria [91]. 


\section{References}

[1] "CDC Morbidity and Mortality Weekly Report "” 53, 57-59, 2004.

[2] D. M. Ferriero, "Neonatal brain injury," The New England Journal of Medicine, vol. 351, pp. 1985-1995, 2004.

[3] S. Niermeyer, J. Kattwinkel, P. Van Reempts et al., "International guidelines for neonatal resuscitation: an excerpt from the guidelines 2000 for cardiopulmonary resuscitation and emergency cardiovascular care: international consensus on science. Contributors and reviewers for the neonatal resuscitation guidelines," Pediatrics, vol. 106, no. 3, p. E29, 2000.

[4] O. D. Saugstad, T. Rootwelt, and O. Aalen, "Resuscitation of asphyxiated newborn infants with room air or oxygen: an international controlled trial: the Resair 2 study," Pediatrics, vol. 102, no. 1, article e1, 1998.

[5] A. Tan, A. Schulze, C. P. O’Donnell, and P. G. Davis, "Air versus oxygen for resuscitation of infants at birth," Cochrane Database of Systematic Reviews, no. 3, Article ID CD002273, 2005.

[6] M. Vento and O. D. Saugstad, "Oxygen supplementation in the delivery room: updated information," Journal of Pediatrics, vol. 158, supplement 2, pp. e5-e7, 2011.

[7] M. Vento, M. Asensi, J. Sastre, A. Lloret, F. García-Sala, and J. Viña, "Oxidative stress in asphyxiated term infants resuscitated with $100 \%$ oxygen," Journal of Pediatrics, vol. 142, no. 3, pp. 240-246, 2003.

[8] J. D. Koch, D. K. Miles, J. A. Gilley, C. P. Yang, and S. G. Kernie, "Brief exposure to hyperoxia depletes the glial progenitor pool and impairs functional recovery after hypoxic-ischemic brain injury," Journal of Cerebral Blood Flow and Metabolism, vol. 28, no. 7, pp. 1294-1306, 2008.

[9] E. Haase, D. L. Bigam, Q. B. Nakonechny, L. D. Jewell, G. Korbutt, and P. Y. Cheung, "Resuscitation with 100\% oxygen causes intestinal glutathione oxidation and reoxygenation injury in asphyxiated newborn piglets," Annals of Surgery, vol. 240, no. 2, pp. 364-373, 2004.

[10] B. H. Munkeby, W. B. Børke, K. Bjørnland et al., "Resuscitation with $100 \% \mathrm{O}_{2}$ increases cerebral injury in hypoxemic piglets," Pediatric Research, vol. 56, no. 5, pp. 783-790, 2004.

[11] K. Van Meter, S. Sheps, F. Kriedt et al., "Hyperbaric oxygen improves rate of return of spontaneous circulation after prolonged normothermic porcine cardiopulmonary arrest," Resuscitation, vol. 78, no. 2, pp. 200-214, 2008.

[12] R. Linner, O. Werner, V. Perez-De-Sa, and D. Cunha-Goncalves, "Circulatory recovery is as fast with air ventilation as with 100\% oxygen after asphyxia-induced cardiac arrest in piglets," Pediatric Research, vol. 66, no. 4, pp. 391-394, 2009.

[13] A. L. Solevåg, I. Dannevig, B. Nakstad, and O. D. Saugstad, "Resuscitation of severely asphyctic newborn pigs with cardiac arrest by using $21 \%$ or $100 \%$ oxygen," Neonatology, vol. 98 , no. 1 , pp. $64-72,2010$.

[14] D. Matsiukevich, T. M. Randis, I. Utkina-Sosunova, R. A. Polin, and V. S. Ten, "The state of systemic circulation, collapsed or preserved defines the need for hyperoxic or normoxic resuscitation in neonatal mice with hypoxia-ischemia," Resuscitation, vol. 81, no. 2, pp. 224-229, 2010.

[15] J. S. Ditelberg, R. A. Sheldon, C. J. Epstein, and D. M. Ferriero, "Brain injury after perinatal hypoxia-ischemia is exacerbated in copper/zinc superoxide dismutase transgenic mice," Pediatric Research, vol. 39, no. 2, pp. 204-208, 1996.

[16] R. A. Sheldon, S. Christen, and D. M. Ferriero, "Genetic and pharmacologic manipulation of oxidative stress after neonatal hypoxia-ischemia," International Journal of Developmental Neuroscience, vol. 26, no. 1, pp. 87-92, 2008.
[17] A. Y. Abramov, A. Scorziello, and M. R. Duchen, "Three distinct mechanisms generate oxygen free radicals in neurons and contribute to cell death during anoxia and reoxygenation," Journal of Neuroscience, vol. 27, no. 5, pp. 1129-1138, 2007.

[18] O. D. Saugstad and L. Gluck, "Plasma hypoxanthine levels in newborn infants: a specific indicator of hypoxia," Journal of Perinatal Medicine, vol. 10, no. 6, pp. 266-272, 1982.

[19] O. D. Saugstad, "Hypoxanthine as an indicator of hypoxia: its role in health and disease through free radical production," Pediatric Research, vol. 23, no. 2, pp. 143-150, 1988.

[20] Y. Feng, W. Shi, M. Huang, and M. H. LeBlanc, "Oxypurinol administration fails to prevent hypoxic-ischemic brain injury in neonatal rats," Brain Research Bulletin, vol. 59, no. 6, pp. 453-457, 2003.

[21] T. Chaudhari and W. McGuire, "Allopurinol for preventing mortality and morbidity in newborn infants with suspected hypoxic-ischaemic encephalopathy," Cochrane Database of Systematic Reviews, no. 2, Article ID CD006817, 2008.

[22] C. Doverhag, M. Keller, A. Karlsson et al., "Pharmacological and genetic inhibition of NADPH oxidase does not reduce brain damage in different models of perinatal brain injury in newborn mice," Neurobiology of Disease, vol. 31, no. 1, pp. 133-144, 2008.

[23] G. Loor, J. Kondapalli, H. Iwase et al., "Mitochondrial oxidant stress triggers cell death in simulated ischemia-reperfusion," Biochimica et Biophysica Acta, vol. 1813, no. 7, pp. 1382-1394, 2011.

[24] A. A. Starkov, C. Chinopoulos, and G. Fiskum, "Mitochondrial calcium and oxidative stress as mediators of ischemic brain injury," Cell Calcium, vol. 36, no. 3-4, pp. 257-264, 2004.

[25] G. Ambrosio, J. L. Zweier, C. Duilio et al., "Evidence that mitochondrial respiration is a source of potentially toxic oxygen free radicals in intact rabbit hearts subjected to ischemia and reflow," Journal of Biological Chemistry, vol. 268, no. 25, pp. 18532-18541, 1993.

[26] Q. Chen, S. Moghaddas, C. L. Hoppel, and E. J. Lesnefsky, "Ischemic defects in the electron transport chain increase the production of reactive oxygen species from isolated rat heart mitochondria," American Journal of Physiology-Cell Physiology, vol. 294, no. 2, pp. C460-C466, 2008.

[27] C. A. Piantadosi and J. Zhang, "Mitochondrial generation of reactive oxygen species after brain ischemia in the rat," Stroke, vol. 27, no. 2, pp. 327-332, 1996.

[28] M. G. Perrelli, P. Pagliaro, and C. Penna, "Ischemia/reperfusion injury and cardioprotective mechanisms: role of mitochondria and reactive oxygen species," World Journal of Cardiology, vol. 3, no. 6, pp. 186-200, 2011.

[29] M. Simerabet, E. Robin, I. Aristi et al., "Preconditioning by an in situ administration of hydrogen peroxide: involvement of reactive oxygen species and mitochondrial ATP-dependent potassium channel in a cerebral ischemia-reperfusion model," Brain Research, vol. 1240, pp. 177-184, 2008.

[30] M. V. Cohen, X. M. Yang, and J. M. Downey, "Acidosis, oxygen, and interference with mitochondrial permeability transition pore formation in the early minutes of reperfusion are critical to postconditioning's success," Basic Research in Cardiology, vol. 103, no. 5, pp. 464-471, 2008.

[31] V. S. Ten, J. Yao, V. Ratner et al., "Complement component C1q mediates mitochondria-driven oxidative stress in neonatal hypoxic-ischemic brain injury," Journal of Neuroscience, vol. 30, no. 6, pp. 2077-2087, 2010.

[32] Q. Chen, E. J. Vazquez, S. Moghaddas, C. L. Hoppel, and E. J. Lesnefsky, "Production of reactive oxygen species 
by mitochondria: central role of complex III," Journal of Biological Chemistry, vol. 278, no. 38, pp. 36027-36031, 2003.

[33] M. V. Skulachev, Y. N. Antonenko, V. N. Anisimov et al., "Mitochondrial-targeted plastoquinone derivatives. Effect on senescence and acute age-related pathologies," Current Drug Targets, vol. 12, no. 6, pp. 800-826, 2011.

[34] H. H. Szeto, S. Liu, Y. Soong et al., "Mitochondria-targeted peptide accelerates ATP recovery and reduces ischemic kidney injury," Journal of the American Society of Nephrology, vol. 22, no. 6, pp. 1041-1052, 2011.

[35] M. Rocha, A. Hernandez-Mijares, K. Garcia-Malpartida, C. Bañuls, L. Bellod, and V. M. Victor, "Mitochondria-targeted antioxidant peptides," Current Pharmaceutical Design, vol. 16, no. 28, pp. 3124-3131, 2010.

[36] K. Choi, J. Kim, G. W. Kim, and C. Choi, "Oxidative stressinduced necrotic cell death via mitochondira-dependent burst of reactive oxygen species," Current Neurovascular Research, vol. 6, no. 4, pp. 213-222, 2009.

[37] E. J. Lesnefsky, Q. Chen, S. Moghaddas, M. O. Hassan, B. Tandler, and C. L. Hoppel, "Blockade of electron transport during ischemia protects cardiac mitochondria," Journal of Biological Chemistry, vol. 279, no. 46, pp. 47961-47967, 2004.

[38] A. J. Tompkins, L. S. Burwell, S. B. Digerness, C. Zaragoza, W. L. Holman, and P. S. Brookes, "Mitochondrial dysfunction in cardiac ischemia-reperfusion injury: ROS from complex I, without inhibition," Biochimica et Biophysica Acta, vol. 1762, no. 2, pp. 223-231, 2006.

[39] A. A. Starkov, "The role of mitochondria in reactive oxygen species metabolism and signaling," Annals of the New York Academy of Sciences, vol. 1147, pp. 37-52, 2008.

[40] A. Y. Andreyev, Y. E. Kushnareva, and A. A. Starkov, "Mitochondrial metabolism of reactive oxygen species," Biochemistry, vol. 70, no. 2, pp. 200-214, 2005.

[41] A. A. Starkov, G. Fiskum, C. Chinopoulos et al., "Mitochondrial $\alpha$-ketoglutarate dehydrogenase complex generates reactive oxygen species," Journal of Neuroscience, vol. 24, no. 36, pp. 7779-7788, 2004.

[42] L. Tretter and V. Adam-Vizi, "Generation of reactive oxygen species in the reaction catalyzed by $\alpha$-ketoglutarate dehydrogenase," Journal of Neuroscience, vol. 24, no. 36, pp. 7771-7778, 2004.

[43] E. B. Tahara, F. D. T. Navarete, and A. J. Kowaltowski, “Tissue-, substrate-, and site-specific characteristics of mitochondrial reactive oxygen species generation," Free Radical Biology and Medicine, vol. 46, no. 9, pp. 1283-1297, 2009.

[44] A. Almeida, K. L. Allen, T. E. Bates, and J. B. Clark, "Effect of reperfusion following cerebral ischaemia on the activity of the mitochondrial respiratory chain in the gerbil brain," Journal of Neurochemistry, vol. 65, no. 4, pp. 1698-1703, 1995.

[45] K. L. Allen, A. Almeida, T. E. Bates, and J. B. Clark, "Changes of respiratory chain activity in mitochondrial and synaptosomal fractions isolated from the gerbil brain after graded ischaemia," Journal of Neurochemistry, vol. 64, no. 5, pp. 2222-2229, 1995.

[46] E. Gilland, M. Puka-Sundvall, L. Hillered, and H. Hagberg, "Mitochondrial function and energy metabolism after hypoxia-ischemia in the immature rat brain: involvement of NMDA-receptors," Journal of Cerebral Blood Flow and Metabolism, vol. 18, no. 3, pp. 297-304, 1998.

[47] N. R. Sims, "Selective impairment of respiration in mitochondria isolated from brain subregions following transient forebrain ischemia in the rat," Journal of Neurochemistry, vol. 56, no. 6, pp. 1836-1844, 1991.
[48] J. Folbergrova, B. Ljunggren, K. Norberg, and B. K. Siesjo, "Influence of complete ischemia on glycolytic metabolites, citric acid cycle intermediates, and associated amino acids in the rat cerebral cortex," Brain Research, vol. 80, no. 2, pp. 265-279, 1974.

[49] G. Benzi, E. Arrigoni, F. Marzatico, and R. F. Villa, "Influence of some biological pyrimidines on the succinate cycle during and after cerebral ischemia," Biochemical Pharmacology, vol. 28, no. 17, pp. 2545-2550, 1979.

[50] V. A. Khazanov, A. N. Poborsky, and M. N. Kondrashova, "Air saturation of the medium reduces the rate of phosphorylating oxidation of succinate in isolated mitochondria," FEBS Letters, vol. 314, no. 3, pp. 264-266, 1992.

[51] A. N. Poborskii, "Effect of research conditions on succinate oxidation in brain mitochondria in circulatory hypoxia," Patologicheskaia Fiziologiia i Eksperimental'naia Terapiia's, no. 1, pp. 10-12, 1997.

[52] T. König, D. G. Nicholls, and P. B. Garland, "The inhibition of pyruvate and Ls(+)-isocitrate oxidation by succinate oxidation in rat liver mitochondria," Biochemical Journal, vol. 114, no. 3, pp. 589-596, 1969.

[53] O. Pisarenko, I. Studneva, and V. Khlopkov, "Metabolism of the tricarboxylic acid cycle intermediates and related amino acids in ischemic guinea pig heart," Biomedica Biochimica Acta, vol. 46, no. 8-9, pp. S568-S571, 1987.

[54] K. Kato, T. Matsubara, and N. Sakamoto, "Correlation between myocardial blood flow and tissue succinate during acute ischemia," Nagoya Journal of Medical Science, vol. 57, no. 1-4, pp. 43-50, 1994.

[55] Z. V. Niatsetskaya, S. A. Sosunov, D. Matsiukevich et al., "The oxygen free radicals originating from mitochondrial complex I contribute to oxidative brain injury following hypoxia-ischemia in neonatal mice," Journal of Neuroscience, vol. 32, no. 9, pp. 3235-3244, 2012.

[56] F. Han, T. Da, N. A. Riobo, and L. B. Becker, "Early mitochondrial dysfunction in electron transfer activity and reactive oxygen species generation after cardiac arrest," Critical Care Medicine, vol. 36, supplement 11, pp. S447-S453, 2008.

[57] R. D. Guzy, M. M. Mack, and P. T. Schumacker, "Mitochondrial complex III is required for hypoxia-induced ROS production and gene transcription in yeast," Antioxidants and Redox Signaling, vol. 9, no. 9, pp. 1317-1328, 2007.

[58] I. Sipos, L. Tretter, and V. Adam-Vizi, "The production of reactive oxygen species in intact isolated nerve terminals is independent of the mitochondrial membrane potential," Neurochemical Research, vol. 28, no. 10, pp. 1575-1581, 2003.

[59] J. W. McDonald and M. V. Johnston, "Physiological and pathophysiological roles of excitatory amino acids during cental nervous system development," Brain Research Reviews, vol. 15 , no. 1, pp. 41-70, 1990.

[60] S. L. Tahraoui, S. Marret, C. Bodénant et al., "Central role of microglia in neonatal excitotoxic lesions of the murine periventricular white matter," Brain Pathology, vol. 11, no. 1, pp. 56-71, 2001.

[61] A. A. Starkov, "The molecular identity of the mitochondrial Ca2+ sequestration system: minireview," FEBS Journal, vol. 277, no. 18, pp. 3652-3663, 2010.

[62] S. Matsumoto, H. Friberg, M. Ferrand-Drake, and T. Wieloch, "Blockade of the mitochondrial permeability transition pore diminishes infarct size in the rat after transient middle cerebral artery occlusion," Journal of Cerebral Blood Flow and Metabolism, vol. 19, no. 7, pp. 736-741, 1999.

[63] L. Khaspekov, H. Friberg, A. Halestrap, I. Viktorov, and T. Wieloch, "Cyclosporin A and its nonimmunosuppressive 
analogue $\mathrm{N}-\mathrm{Me}$-Val-4-cyclosporin A mitigate glucose/oxygen deprivation-induced damage to rat cultured hippocampal neurons," European Journal of Neuroscience, vol. 11, no. 9, pp. 3194-3198, 1999.

[64] A. W. C. Leung and A. P. Halestrap, "Recent progress in elucidating the molecular mechanism of the mitochondrial permeability transition pore," Biochimica et Biophysica Acta, vol. 1777, no. 7-8, pp. 946-952, 2008.

[65] F. Di Lisa, N. Kaludercic, A. Carpi, R. Menabò, and M. Giorgio, "Mitochondrial pathways for ROS formation and myocardial injury: the relevance of p66Shc and monoamine oxidase," Basic Research in Cardiology, vol. 104, no. 2, pp. 131-139, 2009.

[66] J. J. Lemasters, T. P. Theruvath, Z. Zhong, and A. L. Nieminen, "Mitochondrial calcium and the permeability transition in cell death," Biochimica et Biophysica Acta, vol. 1787, no. 11, pp. 1395-1401, 2009.

[67] E. Basso, L. Fante, J. Fowlkes, V. Petronilli, M. A. Forte, and P. Bernardi, "Properties of the permeability transition pore in mitochondria devoid of cyclophilin D," Journal of Biological Chemistry, vol. 280, no. 19, pp. 18558-18561, 2005.

[68] J. S. Kim, Y. Jin, and J. J. Lemasters, "Reactive oxygen species, but not $\mathrm{Ca} 2+$ overloading, trigger $\mathrm{pH}$ - and mitochondrial permeability transition-dependent death of adult rat myocytes after ischemia-reperfusion," American Journal of Physiology-Heart and Circulatory Physiology, vol. 290, no. 5, pp. H2024-H2034, 2006.

[69] H. L. Liang, F. Sedlic, Z. Bosnjak, and V. Nilakantan, "SOD1 and MitoTEMPO partially prevent mitochondrial permeability transition pore opening, necrosis, and mitochondrial apoptosis after ATP depletion recovery," Free Radical Biology and Medicine, vol. 49, no. 10, pp. 1550-1560, 2010.

[70] M. Puka-Sundvall, C. Wallin, E. Gilland et al., "Impairment of mitochondrial respiration after cerebral hypoxia-ischemia in immature rats: relationship to activation of caspase-3 and neuronal injury," Developmental Brain Research, vol. 125, no. 1-2, pp. 43-50, 2000.

[71] C. S. Caspersen, A. Sosunov, I. Utkina-Sosunova, V. I. Ratner, A. A. Starkov, and V. S. Ten, "An isolation method for assessment of brain mitochondria function in neonatal mice with hypoxic-ischemic brain injury," Developmental Neuroscience, vol. 30, no. 5, pp. 319-324, 2008.

[72] A. Vinogradov, A. Scarpa, and B. Chance, "Calcium and pyridine nucleotide interaction in mitochondrial membranes," Archives of Biochemistry and Biophysics, vol. 152, no. 2, pp. 646-654, 1972.

[73] C. P. Baines, R. A. Kaiser, N. H. Purcell et al., "Loss of cyclophilin D reveals a critical role for mitochondrial permeability transition in cell death," Nature, vol. 434, no. 7033, pp. 658-662, 2005.

[74] T. Nakagawa, S. Shimizu, T. Watanabe et al., "Cyclophilin D-dependent mitochondrial permeability transition regulates some necrotic but not apoptotic cell death," Nature, vol. 434, no. 7033, pp. 652-658, 2005.

[75] M. Puka-Sundvall, B. Gajkowska, M. Cholewinski, K. Blomgren, J. W. Lazarewicz, and H. Hagberg, "Subcellular distribution of calcium and ultrastructural changes after cerebral hypoxia-ischemia in immature rats," Developmental Brain Research, vol. 125, no. 1-2, pp. 31-41, 2000.

[76] X. Wang, Y. Carlsson, E. Basso et al., "Developmental shift of cyclophilin D contribution to hypoxic-ischemic brain injury," Journal of Neuroscience, vol. 29, no. 8, pp. 2588-2596, 2009.

[77] M. Puka-Sundvall, E. Gilland, and H. Hagberg, "Cerebral hypoxia-ischemia in immature rats: involvement of mitochondrial permeability transition?" Developmental Neuroscience, vol. 23, no. 3, pp. 192-197, 2001.

[78] J. H. Hwang, J. H. Lee, K. H. Lee et al., "Cyclosporine A attenuates hypoxic-ischemic brain injury in newborn rats," Brain Research, vol. 1359, pp. 208-215, 2010.

[79] P. L. Leger, D. De Paulis, S. Branco et al., "Evaluation of cyclosporine A in a stroke model in the immature rat brain," Experimental Neurology, vol. 230, no. 1, pp. 58-66, 2011.

[80] X. D. Liu, G. Y. Pan, L. Xie et al., "Cyclosporin A enhanced protection of nimodipine against brain damage induced by hypoxia-ischemia in mice and rats," Acta Pharmacologica Sinica, vol. 23, no. 3, pp. 225-229, 2002.

[81] I. Marzo, C. Brenner, N. Zamzami et al., "Bax and adenine nucleotide translocator cooperate in the mitochondrial control of apoptosis," Science, vol. 281, no. 5385, pp. 20272031, 1998.

[82] R. Eskes, B. Antonsson, A. Osen-Sand et al., "Bax-induced cytochrome $\mathrm{C}$ release from mitochondria is independent of the permeability transition pore but highly dependent on Mg2+ ions," Journal of Cell Biology, vol. 143, no. 1, pp. 217-224, 1998.

[83] W. Korytowski, L. V. Basova, A. Pilat, R. M. Kernstock, and A. W. Girotti, "Permeabilization of the mitochondrial outer membrane by Bax/truncated Bid (tBid) proteins as sensitized by cardiolipin hydroperoxide translocation: mechanistic implications for the intrinsic pathway of oxidative apoptosis," The Journal of Biological Chemistry, vol. 286, no. 30, pp. 26334-26343, 2011.

[84] E. Jacotot, P. Costantini, E. Laboureau, N. Zamzami, S. A. Susin, and G. Kroemer, "Mitochondrial membrane permeabilization during the apoptotic process," Annals of the New York Academy of Sciences, vol. 887, pp. 18-30, 1999.

[85] H. Hagberg, C. Mallard, C. I. Rousset Catherine, and X. Wang, "Apoptotic mechanisms in the immature brain: involvement of mitochondria," Journal of Child Neurology, vol. 24, no. 9, pp. 1141-1146, 2009.

[86] X. Wang, W. Han, X. Du et al., "Neuroprotective effect of Bax-inhibiting peptide on neonatal brain injury," Stroke, vol. 41, no. 9, pp. 2050-2055, 2010.

[87] P. Pasdois, J. E. Parker, E. J. Griffiths, and A. P. Halestrap, “The role of oxidized cytochrome $\mathrm{c}$ in regulating mitochondrial reactive oxygen species production and its perturbation in ischaemia," Biochemical Journal, vol. 436, no. 2, pp. 493-505, 2011.

[88] L. Vela, M. Contel, L. Palomera, G. Azaceta, and I. Marzo, "Iminophosphorane-organogold (III) complexes induce cell death through mitochondrial ROS production," Journal of Inorganic Biochemistry, vol. 105, no. 10, pp. 1306-1313, 2011.

[89] E. R. Hahm, M. B. Moura, E. E. Kelley, B. Van Houten, S. Shiva, and S. V. Singh, "Withaferin a-induced apoptosis in human breast cancer cells is mediated by reactive oxygen species," PLoS One, vol. 6, no. 8, article e23354, 2011.

[90] Q. Ma, H. Fang, W. Shang et al., "Superoxide flashes: early mitochondrial signals for oxidative stress-induced apoptosis," The Journal of Biological Chemistry, vol. 286, no. 31, pp. 27573-27581, 2011.

[91] M. Narita, S. Shimizu, T. Ito et al., "Bax interacts with the permeability transition pore to induce permeability transition and cytochrome c release in isolated mitochondria," Proceedings of the National Academy of Sciences of the United States of America, vol. 95, no. 25, pp. 14681-14686, 1998. 


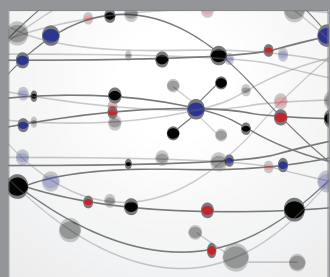

The Scientific World Journal
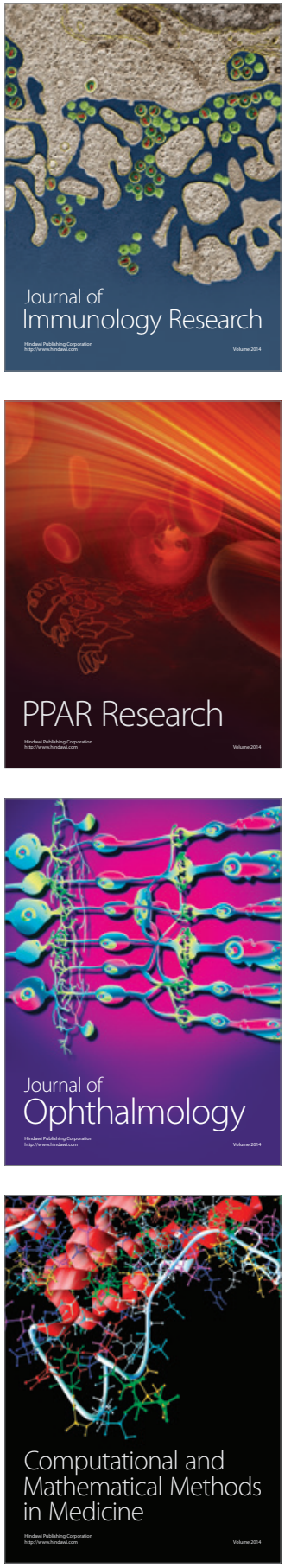

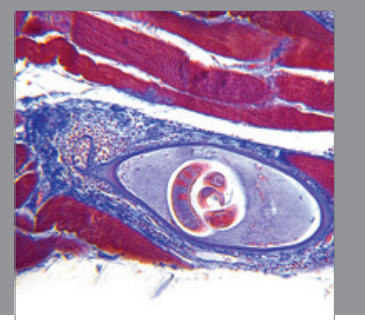

Gastroenterology

Research and Practice
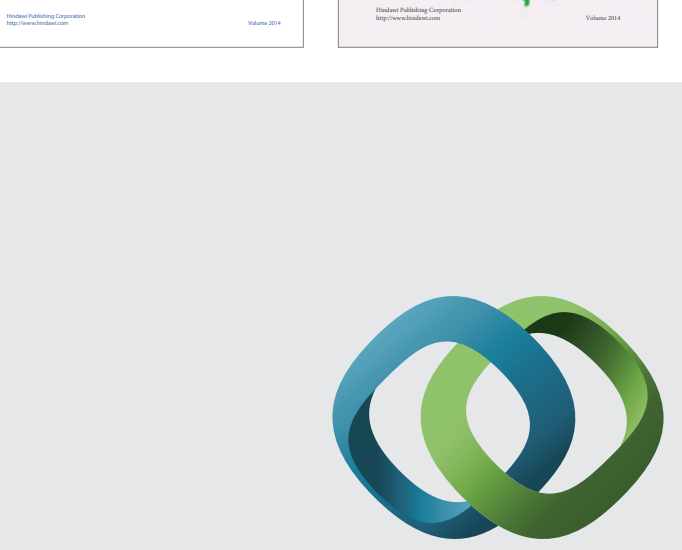

\section{Hindawi}

Submit your manuscripts at

http://www.hindawi.com
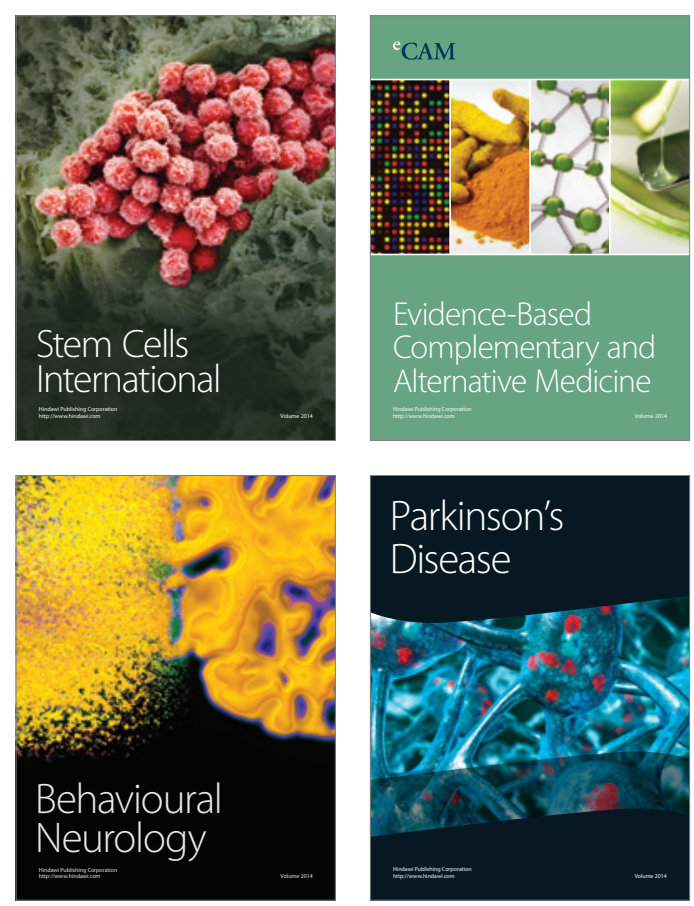

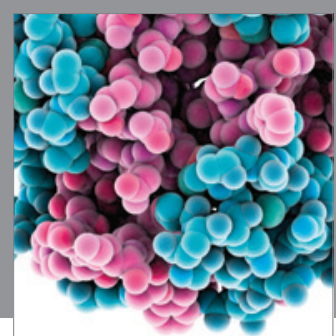

Journal of
Diabetes Research

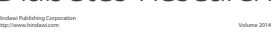

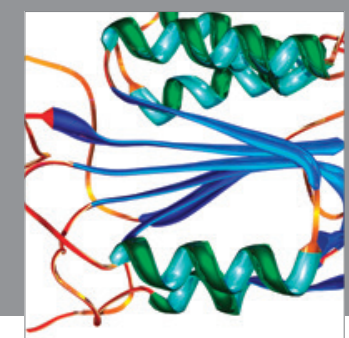

Disease Markers
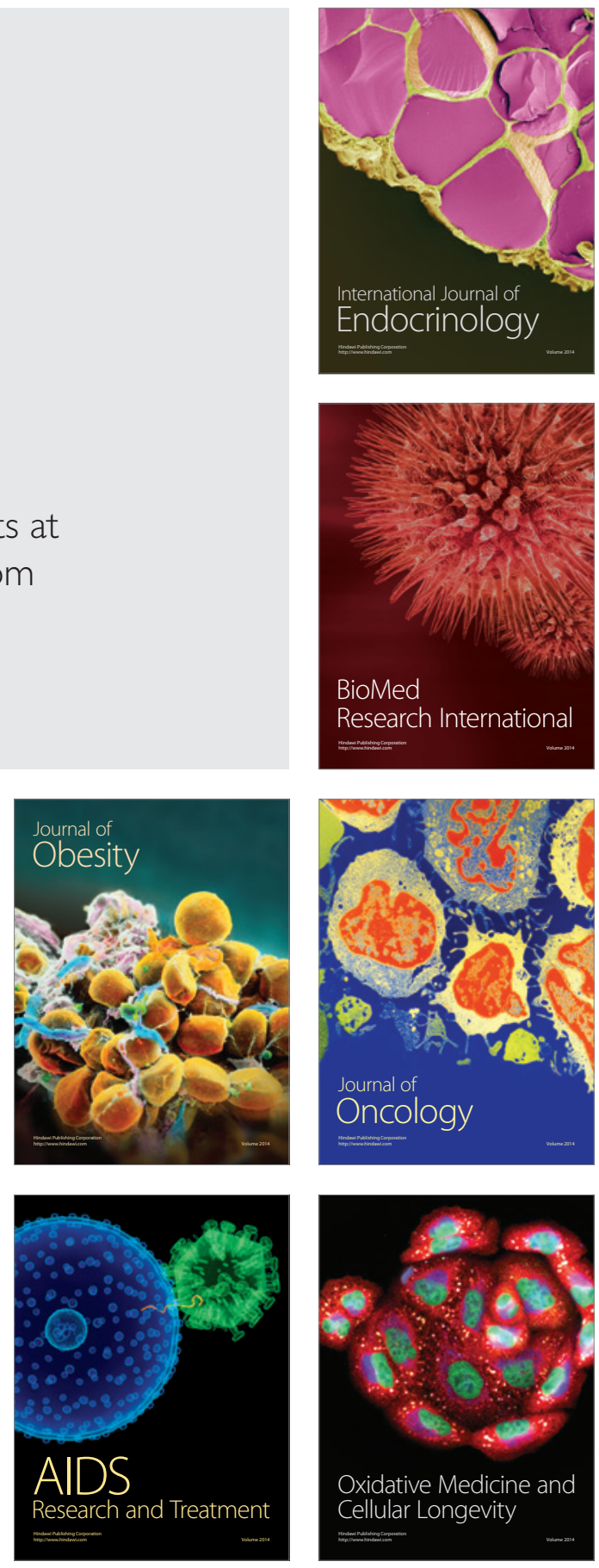